\title{
(6) OPEN ACCESS \\ Experience feedback committee in emergency medicine: a tool for security management
}

\author{
André Lecoanet, ${ }^{1}$ Elodie Sellier, ${ }^{1,2}$ Françoise Carpentier, ${ }^{3}$ Maxime Maignan, ${ }^{3}$ \\ Arnaud Seigneurin, ${ }^{1}$ Patrice François ${ }^{1,2}$
}

${ }^{1}$ Pôle Santé Publique, Unité d'évaluation médicale, Centre Hospitalier Universitaire,

Grenoble, France

${ }^{2}$ UJF-Grenoble 1/CNRS/TIMC

IMAG UMR 5525, Grenoble, France

${ }^{3}$ Département des urgences, Centre Hospitalier Universitaire, Grenoble, France

\section{Correspondence to}

Dr Elodie Sellier, Pôle Santé Publique, Unité d'évaluation médicale, Centre Hospitalier Universitaire, CS10217,

Grenoble F-38043, cedex 9, France;

esellier@chu-grenoble.fr

Received 25 April 2013 Revised 9 July 2013 Accepted 18 July 2013 Published Online First 20 August 2013

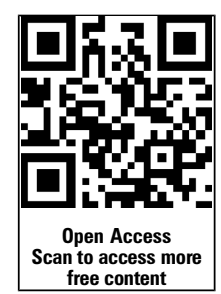

\section{ABSTRACT}

Objective Emergency departments are high-risk structures. The objective was to analyse the functioning of an experience feedback committee (EFC), a security management tool for the analysis of incidents in a medical department.

Methods We conducted a descriptive study based on the analysis of the written documents produced by the EFC between November 2009 and May 2012. We performed a double analysis of all incident reports, meeting minutes and analysis reports.

Results During the study period, there were 22 meetings attended by 15 professionals. 471 reported incidents were transmitted to the EFC. Most of them (95\%) had no consequence for the patients. Only one reported incident led to the patient's death. 12 incidents were analysed thoroughly and the committee decided to set up 14 corrective actions, including eight guideline writing actions, two staff trainings, two resource materials provisions and two organisational changes. Conclusions The staff took part actively in the EFC. Following the analysis of incidents, the EFC was able to set up actions at the departmental level. Thus, an EFC seems to be an appropriate security management tool for an emergency department.

\section{INTRODUCTION}

Considerable attention has been drawn to patient safety since the publication of several studies that reported rates of adverse events ranging between $4 \%$ and $16 \%$ of hospitalised patients. ${ }^{1-4}$ These events were associated with permanent impairment, including death, in $14-41 \%$ of cases. Of these events, $27-51 \%$ were considered preventable, that is, were the result of medical error and would not have occurred with standard medical care. ${ }^{5}$ In emergency departments (EDs), similar rates of adverse events or errors have been observed ${ }^{6-9}$ but with a higher proportion of preventable events $\left(53-80 \% \%^{3} \quad 6 \quad 89\right)$. EDs are high-incident-risk structures, as they treat patients presenting with diverse diseases of extreme severities and for whom historical information is often lacking. ${ }^{10}{ }^{11}$ Moreover, professionals in EDs experience irregular workloads, crowding, ${ }^{11} 12$ disrupted sleep cycles, and numerous interruptions in their work. ${ }^{13}$ Fortunately, the majority of errors do not result in adverse outcomes for patients. Indeed, in their study describing errors in a busy ED, Fordyce et $a l^{7}$ reported that 7 out of 346 errors (2\%) result in a significant adverse outcome.

Several methods of error prevention in EDs have been developed. These methods have focused on teamwork and on reducing the number of drug errors thanks to the intervention of pharmacists or the use of computerised order entry systems. ${ }^{14}$ Ten years ago, specific structures, called experience feedback committees (EFCs), were created to analyse errors or near-miss events within a medical department. Originating from civil aviation security systems, the method has been adapted to healthcare facilities in France with the help of Air France Consulting. ${ }^{15}{ }^{16}$ An EFC is a multidisciplinary team representing the diversity of the functions encountered in the medical unit. The EFC members meet regularly to examine reported incidents related to their medical unit. They choose priority incidents that need to be analysed and propose corrective actions. The main principles of the method are that patient safety must be managed within a medical team, the team must focus on near-miss events, and the actions must concern latent factors that have the potential to cause an adverse event. ${ }^{17}$

The objective of the study was to describe the functioning of the EFC in an ED, to discuss its contribution to the management of quality and patient safety and to evaluate whether such a system would be feasible in any ED setting.

\section{METHODS}

\section{Study design}

This was a descriptive study based on written reports of the ED EFC from its inception in November 2009 until May 2012.

\section{Setting}

The study was conducted in a 1347-bed acute-care university hospital in France, including 20 beds for the ED. The ED has an annual patient volume of 80 000-90000 visits.

The hospital has a voluntary internal reporting system for adverse events and near-misses. The incidents are reported to the central safety unit, from the health surveillance department, in a standardised written document. This safety unit is independent of the EFCs and comprises a medical doctor, a pharmacist and a quality engineer. They receive all of the hospital's reported incidents. They classify them and analyse the most serious incidents or those involving several hospital units, and they notify the different vigilance units when necessary. A vigilance unit is a medical unit that is in charge of a particular risk such as blood products (haemovigilance), medicines (pharmacovigilance) or medical devices. They gather all the incidents concerning the different medical departments managing an EFC and send them the incident reports before the meetings. At the time of 
this study, 21 medical departments, including the ED, were managing an EFC. A particularity of the ED's EFC in comparison with other EFCs in the other departments was that staff could also report incidents directly to the EFC via a simpler form through an allocated box in the unit (such incidents were not systematically reported to the central safety unit).

In this setting, the EFC is supposed to follow the procedure set up in accordance with the method proposed by Air France Consulting. ${ }^{18}$ The EFC is composed of diverse professionals and the meetings are conducted according to a standardised plan: (1) reading the list of incidents reported in the previous month, (2) choosing a priority incident to analyse during the following month, (3) choosing the professional responsible for the analysis, (4) listening to the analysis report from the previous month, (5) choosing corrective actions, and (6) monitoring ongoing actions. The analysis is performed during the month following the EFC by a designated person. The person in charge of the analysis analyses the incident according to the ORION method. ${ }^{17}$ This method has six main steps.

Step 1 Collecting data. The professional responsible for the analysis collects relevant information concerning the incident using debriefing meetings, interviews, document analysis or any other necessary means.

Step 2 Describing the chronological facts occurring before, during and after the incident. From each fact mentioned, the professional has to determine whether guidelines for good practice exist at the department, hospital or national level and if there is a gap between the facts and these guidelines. Chronological facts, existing guidelines and identified errors have to be described.

Step 3 Describing the causes of errors (ie, gaps between guidelines and facts) that are sought in different areas: political, organisational, working conditions, team functioning, procedures, actors and the patient.

Step 4 Looking for and describing the latent factors that could have contributed to the occurrence of the errors. These latent factors are sought in the same areas as the causes of errors. However, their links to the incident are less tangible.

Step 5 Setting up corrective actions. The aim is to reduce the risk of adverse events by correcting latent factors contributing to them.

Step 6 Writing a report of the analysis.

\section{Data collection}

All written documents from the EFC of the ED were analysed. For the purposes of this study, reported incidents were classified according to the department that had reported the incident, the type of incident and the consequence for the patient using the International Classification for Patient Safety. ${ }^{19}$ Written reports from meetings were analysed using a standardised procedure that followed the steps of an EFC analysis (as described above). Analysis reports were analysed using a standardised procedure that followed the steps of the ORION method. The analysis of all documents was performed by two independent investigators. Differences in rating were discussed until a consensus was reached.

\section{Statistical analysis}

Qualitative data are reported as numbers and percentages. Quantitative data are reported as medians and IQR.

\section{RESULTS}

The EFC was set up based on a call for volunteers among the staff with the obligation of having one or two participants for each professional category. Once the multidisciplinary team was constituted, several training sessions were held to teach professionals about the culture of safety and the method to follow. Report templates were provided to the committee. Volunteers committed to participating in the EFC for at least one complete year. At the end of each year, a new call for volunteers was made to replace those who decided to stop.

The committee set up 22 meetings during the study period. A total of 15 medical and paramedical professionals participated in the EFC (table 1). The median number of attendants was 8 (IQR 6-9) per meeting. Minutes were taken for all meetings. Priority events were chosen, analysis reports were presented and actions were decided in more than half of the meetings (table 1). In 13 meetings there was no monitoring of the previous actions (59.1\%).

A total of 471 reported incidents were transmitted to the EFC (table 2). Among them, 101 reports (21.4\%) were communicated directly to the EFC through the devoted box. A median number of 14 incidents (IQR 10-18) were discussed per meeting. Incidents were mainly $(68.2 \%)$ reported by a professional of the department, and $84 \%$ of incidents occurred within the department. Reported incidents were mainly about clinical administration (including incidents in patient identification, patient transfer, admission, discharge) and lack of resources (including beds and staff availability). The majority of incidents had no clinical consequence for the patient (95.1\%, table 2$)$. In five cases, the incident led to moderate harm. This included one delay of care for a patient suffering from a cerebral infarction and four patient falls. One patient fell from a stretcher and broke his nose, one patient fell into a puddle of urine and broke his femur, one patient fell because of a seizure and broke his shoulder, and one patient fell over the bed guardrail and suffered from neurological sequelae. One event led to severe harm

Table 1 Main functioning characteristics of the experience feedback committee of the emergency department

\begin{tabular}{|c|c|c|}
\hline $\begin{array}{l}\text { Participants } \\
\%\end{array}$ & & $\mathrm{~N}=15$ \\
\hline Nurses & 4 & 26.7 \\
\hline Physicians & 3 & 20.0 \\
\hline Head nurses & 2 & 13.3 \\
\hline Hospital porters & 2 & 13.3 \\
\hline Secretary & 1 & 6.7 \\
\hline Quality engineer & 1 & 6.7 \\
\hline Auxiliary nurse & 1 & 6.7 \\
\hline Social worker & 1 & 6.7 \\
\hline Median number of participations per participant (IQR 25-75) & 12 & $(4-16)$ \\
\hline Median number of participants per meeting (IQR 25-75) & 8 & $(6-9)$ \\
\hline Meetings & $\mathrm{N}=22$ & $\%$ \\
\hline Writing of minutes & 22 & 100.0 \\
\hline Listening to the events reported during the previous month & 21 & 95.5 \\
\hline $\begin{array}{l}\text { Choice of a priority event to analyse during the following } \\
\text { month }\end{array}$ & 14 & 63.6 \\
\hline $\begin{array}{l}\text { Listening to the analysis report from the event analysed in the } \\
\text { previous month }\end{array}$ & 13 & 59.1 \\
\hline Decision of actions & 12 & 54.6 \\
\hline Follow-up of previous actions & 9 & 40.9 \\
\hline
\end{tabular}


Table 2 Characteristics of the events reported during the experience feedback committee meetings

\begin{tabular}{|c|c|c|}
\hline Characteristics & $\mathrm{N}=471$ & $\%$ \\
\hline \multicolumn{3}{|l|}{ Incident type } \\
\hline Clinical administration & 151 & 32.1 \\
\hline Resources/organisational management & 107 & 22.7 \\
\hline Clinical process/procedure & 62 & 13.2 \\
\hline Behaviour & 52 & 11.0 \\
\hline Medical device/equipment & 30 & 6.4 \\
\hline Documentation & 22 & 4.7 \\
\hline Infrastructure/building/fixtures & 12 & 2.5 \\
\hline Patient accidents & 10 & 2.1 \\
\hline Healthcare-associated infection & 9 & 1.9 \\
\hline Medication/IV fluids & 6 & 1.3 \\
\hline Nutrition & 4 & 0.8 \\
\hline Blood/blood products & 3 & 0.6 \\
\hline Oxygen/gas/vapour & 3 & 0.6 \\
\hline \multicolumn{3}{|l|}{ Degree of harm } \\
\hline None, without care modification & 407 & 86.4 \\
\hline None, with care modification & 41 & 8.7 \\
\hline Mild & 16 & 3.4 \\
\hline Moderate & 5 & 1.1 \\
\hline Severe & 1 & 0.2 \\
\hline Death & 1 & 0.2 \\
\hline \multicolumn{3}{|l|}{ Report provider } \\
\hline Staff from the emergency department & 321 & 68.2 \\
\hline Staff from another department & 150 & 31.8 \\
\hline \multicolumn{3}{|l|}{ Place of the event } \\
\hline In the emergency department & 398 & 84.5 \\
\hline In another department & 73 & 15.5 \\
\hline
\end{tabular}

for a patient who fell because of a defective stretcher. This fall caused a subarachnoid haemorrhage and cardiac arrest. One incident was judged as contributing to a patient's death: the patient was hospitalised in a corridor while suffering from pulmonary insufficiency. An optimal surveillance was consequently not possible.

Fourteen priority incidents were chosen for investigation, including six related to patient transfer or discharge, four to patient identification errors, three to organisational problem and one to accidental blood exposure. Two chosen incidents were finally not investigated. Among the 12 analyses performed, 10 ended with a written report and in two cases the report was made orally (table 3). The written reports showed that the ORION method was often followed. The chronology of facts, identification of gaps and identification of contributing or latent factors were described in $60 \%$ of cases. However, the description of existing recommendations was made in only one quarter of the cases.

Of the 23 proposed corrective actions, the committee decided to implement 14 ones. The action most commonly performed was written guidelines $(n=8$; see box 1$)$.

\section{DISCUSSION}

The study shows that the EFC of the study ED is routinely functioning. The staff took an active part in the procedure and the initiative seems to be well integrated into the department's routine. Following the meetings, corrective actions were decided and set up. These results suggest that the EFC method can be used as a tool to involve healthcare professionals in patient safety management in emergency units.
Table 3 Characteristics of the analysis reports and of the corrective actions

\begin{tabular}{|c|c|c|}
\hline Analysis reports & $\mathrm{N}=12$ & $\%$ \\
\hline S6: Written reports & 10 & 83.3 \\
\hline Oral reports & 2 & 16.7 \\
\hline S1: Description of the data collection method & 10 & 83.3 \\
\hline Individual interviews & 10 & 100.0 \\
\hline Collective debriefing & 10 & 100.0 \\
\hline Files & 6 & 60.0 \\
\hline Area visits & 4 & 40.0 \\
\hline S2: Description of the chronology of facts & 9 & 75.0 \\
\hline S2: Description of existing recommendations & 3 & 25.0 \\
\hline S2: Error identification & 7 & 58.3 \\
\hline S3 and S4: Identification of contributing or latent factors & 11 & 91.7 \\
\hline Management & 2 & 18.2 \\
\hline Organisation and procedures & 11 & 100.0 \\
\hline Working environment & 7 & 63.6 \\
\hline Teamwork & 7 & 63.6 \\
\hline Technical processes & 6 & 54.5 \\
\hline Professionals & 4 & 36.4 \\
\hline Patients & 3 & 27.3 \\
\hline \multicolumn{3}{|l|}{ Corrective actions } \\
\hline S5: Proposed actions & $\mathrm{N}=23$ & \\
\hline Staff training & 6 & 26.1 \\
\hline Writing procedures & 8 & 34.8 \\
\hline Organisational changes & 5 & 21.7 \\
\hline Increasing resource materials & 4 & 17.4 \\
\hline Decided actions & $\mathrm{N}=14$ & \\
\hline With a professional in charge & 8 & 57.1 \\
\hline From the ED & 7 & 87.5 \\
\hline From another department & 1 & 12.5 \\
\hline With a defined deadline & 6 & 42.9 \\
\hline
\end{tabular}

ED, emergency department.

\section{Box 1 Decided corrective actions}

\section{Guideline writing}

- Blood exposure accident

- Inpatient transfer

- Checklist for inpatient transfer

- Patient discharge

- User manual for the computerised medical record

- Patient medical record management

- Admission in the short-term hospitalisation unit

- Care process in examination and care area

Organisational changes

- Change of patient identity label storage

- Stop the transfers to the ED

Training

- Rules for inpatient transfers in the hospital

- Use of computerised medical records

Resource materials

- Provision of care kit for blood exposure accident

- Implementation of an alert system in the computerised medical record 
Thanks to the ORION method, the EFC used a structured and systemic safety approach to analysing incidents. The principle is to choose only one event per meeting in order to analyse it thoroughly. The choice is based either on the gravity of the event or on the frequency of occurrence. Such an approach, like the protocol of the Association of Litigation and Risk Management (ALARM), aims to identify the latent factors that have contributed to an incident so as to set up errorreduction strategies. ${ }^{20}$ Adverse events are a result of various factors, and therefore analyses of clinical incidents should focus on the system's vulnerabilities ${ }^{21-23}$ rather than on individual errors. In this study, system vulnerabilities were most frequently identified as the potential factors of occurrence of events. Nevertheless, we observed some differences in the way the committee conducted their analyses compared to the guidelines. The analysis did not always follow all the steps of the ORION method. These deviations from the method were probably due to the novelty of this activity in the department, and we cannot exclude a lack of training for the participants as another contributing factor. Indeed, formal training was offered at the start but it involved the professionals who participated initially. Additional training was not requested by the team and not proposed afterwards. Nevertheless, we believe that regular training is necessary to ensure good-quality meetings and event analysis and to ensure formal training for new participants. Consequently, we believe that regular formal training sessions must be part of the establishment of an EFC. Another explanation to the deviations from the method could be that the method is too complicated or too time-consuming to be performed completely by professionals who already have a significant clinical workload. Perhaps steps 4 and 5 could be combined, since it is often difficult to distinguish between a contributing and a latent factor. A mean of one action every 2 months was decided, which was deemed satisfactory. However, monitoring the actions was mentioned in the subsequent written reports in only eight cases (out of the 14 actions decided). In six cases, we did not find written information on whether or not the actions were monitored. Most of the actions concerned organisational aspects of care (eg, an incident during an inpatient transfer led to revising the inpatient transfer guidelines and to writing a checklist). This is in accordance with the aim of the method. Indeed, while mortality and morbidity conferences that are also performed in the ED usually focus on medical practices, ${ }^{24} 25$ the EFC uses a system-wide approach that allows organisational failures to be detected. In most of the cases, these failures do not have an effect on the patient but could potentially do so, which is why it is important to identify the failures and prevent them. ${ }^{26}$

One of the principles of an EFC is to gather all categories of professionals who are involved in the unit. This is one of the major differences with mortality and morbidity conferences, where most often only residents and senior physicians attend. ${ }^{27} 28$ In the ED, professionals from different clinical areas were part of the committee and participated in the analysis and setting up of actions. Multidisciplinary meetings reinforce interprofessional collaboration and communication and they allow all core business partners to be involved in patient safety. Moreover, Kauffman et $a^{29}$ suggested that a multidisciplinary approach contributes towards identifying system vulnerabilities more easily.

Also, the EFC can only function properly if professionals report near-misses or adverse events. Several barriers to incident reporting have been identified such as time constraints, complex forms, fear of punishment, shame, a lack of education and a lack of feedback. ${ }^{30-32}$ However, it is not the objective of the
EFC to treat all near-misses or adverse events. In this study, a median number of 14 incidents were reported and discussed per meeting, which is a sufficient number to choose an incident of interest. Less than $5 \%$ of incidents had a consequence for the patient. This is what is expected by an EFC, as adverse events are preferentially analysed in the morbidity and mortality conference. The objective of the EFC was to preferentially analyse the near-misses as they can potentially cause harm. Finding the causes to the near-misses makes it possible to set up corrective actions before an adverse event actually occurs.

This study had several limitations. First, the functioning of an EFC depends on people who are involved, and this study was performed in only one department. Studying another team in a different context could have other results. Second, we were not able to measure the impact of the EFC on patient safety. However, we suppose that corrective actions against identified vulnerabilities improve patient safety. Also, we suppose that the EFC has an impact of safety culture on all the professionals in the committee.

In conclusion, this study showed that all categories of professionals can take part in an EFC in order to develop actions aiming to increase patient safety. Also, we showed that the recommended way of conducting the EFC was not always followed, suggesting that we should focus on training attendees and devising a way to simplify the method for care professionals who are always short of time. However, corrective actions were taken which proves that the EFC was a success.

Acknowledgements We acknowledge all the professionals who participated in the EFC: Patrice François, Françoise Carpentier, lleana Ciocan, Laure Bourdieu, Dominique Ferley, Annie Bodin, Isabelle Mignon, Frederic Martinot, Salvatrice Mazzilli, Hamoudi Benbakir, Cedric Rouge, Anne-Laure Dravigny, Anne Juillet and Valerie Reynier. We acknowledge Linda Northrup for her assistance in editing the manuscript in English.

Contributors AL: acquisition of the data, analysis and interpretation of the data, drafting the manuscript. ES: analysis and interpretation of the data, drafting the manuscript. FC: acquisition of the data, critical revision of the manuscript. MM: acquisition of the data, critical revision of the manuscript. AS: critical revision of the manuscript for important intellectual content. PF: study concept and design, analysis and interpretation of the data, critical revision of the manuscript, obtained funding, study supervision.

Funding This research was funded by a grant from the French Ministry of Health (PREPS 12-026-0001 2012).

\section{Competing interests None.}

Provenance and peer review Not commissioned; externally peer reviewed.

Open Access This is an Open Access article distributed in accordance with the Creative Commons Attribution Non Commercial (CC BY-NC 3.0) license, which permits others to distribute, remix, adapt, build upon this work non-commercially, and license their derivative works on different terms, provided the original work is properly cited and the use is non-commercial. See: http://creativecommons.org/ licenses/by-nc/3.0/

\section{REFERENCES}

1 Brennan TA, Leape LL, Laird NM, et al. Incidence of adverse events and negligence in hospitalized patients. Results of the Harvard Medical Practice Study I. N Engl J Med 1991:324:370-6.

2 Vincent C, Neale G, Woloshynowych M. Adverse events in British hospitals: preliminary retrospective record review. BMJ 2001;322:517-19.

3 Wilson RM, Runciman WB, Gibberd RW, et al. The Quality in Australian Health Care Study. Med J Aust 1995;163:458-71.

4 Michel $P$, Minodier $C$, Lathelize $M$, et al. Les évènements indésirables graves associés aux soins observés dans les établissements de santé. Dossiers Solidarité et Santé 2010;17:1-18.

5 Kohn LT, Corrigan JM, Donaldson MS, eds. To err is human: building a safer health system. Institute of Medicine Report. Washington, DC: National Academy Press, 1999.

6 Forster AJ, Rose NG, van Walraven C, et al. Adverse events following an emergency department visit. Qual Saf Health Care 2007;16:17-22.

7 Fordyce J, Blank FS, Pekow $\mathrm{P}$, et al. Errors in a busy emergency department. Ann Emerg Med 2003:42:324-33.

8 Thomas EJ, Studdert DM, Burstin HR, et al. Incidence and types of adverse events and negligent care in Utah and Colorado. Medical Care 2000:38:261-71. 
9 Friedman SM, Provan D, Moore $S$, et al. Errors, near misses and adverse events in the emergency department: what can patients tell us? CJEM 2008;10:421-7.

10 Stiell A, Forster AJ, Stiell IG, et al. Prevalence of information gaps in the emergency department and the effect on patient outcomes. CMAJ 2003;169:1023-8.

11 Horwitz LI, Meredith T, Schuur JD, et al. Dropping the baton: a qualitative analysis of failures during the transition from emergency department to inpatient care. Ann Emerg Med 2009;53:701-10.e4.

12 Tang N, Stein J, Hsia RY, et al. Trends and characteristics of US emergency department visits, 1997-2007. JAMA 2010;304:664-70.

13 Chisholm CD, Collison EK, Nelson DR, et al. Emergency department workplace interruptions: are emergency physicians 'interrupt-driven' and 'multitasking'? Acad Emerg Med 2000;7:1239-43.

14 Schenkel S. Promoting patient safety and preventing medical error in emergency departments. Acad Emerg Med 2000;7:1204-22.

15 Lartigau E, Coche-Dequeant B, Dumortier V, et al. [Experience feed back committee in radiotherapy (CREx): a compulsory tool for security management evaluation at two years]. Cancer Radiother 2008;12:610-13.

16 Woynar S, Debouck F, Cellier $\mathrm{P}$, et al. [Towards global security in radiation oncology. Using event notification feed-back]. Cancer Radiother 2007;11:320-8.

17 Debouck F, Rieger E, Petit $\mathrm{H}$, et al. [ORION(R): a simple and effective method for systemic analysis of clinical events and precursors occurring in hospital practice]. Cancer Radiother 2012;16:201-8.

18 Francois $P$, Sellier $E$, Imburchia $F$, et al. [Experience feedback committee: a method for patient safety improvement]. Rev Epidemiol Sante Publique 2013;61:155-61.

19 World Alliance For Patient Safety, Drafting G, Sherman H, Castro G, et al. Towards an International Classification for Patient Safety: the conceptual framework. Int J Qual Health Care 2009;21:2-8.

20 Vincent C, Taylor-Adams S, Chapman EJ, et al. How to investigate and analyse clinical incidents: clinical risk unit and association of litigation and risk management protocol. BMJ 2000;320:777-81
21 Fee $\mathrm{C}$, Hall K, Morrison JB, et al. Consensus-based recommendations for research priorities related to interventions to safeguard patient safety in the crowded emergency department. Acad Emerg Med 2011:18:1283-8.

22 Reason JT, Carthey J, de Leval MR. Diagnosing vulnerable system syndrome: an essential prerequisite to effective risk management. Qual Health Care 2001; 10(Suppl 2):ii21-5.

23 Perry SJ. Profiles in patient safety: organizational barriers to patient safety. Acad Emerg Med 2002;9:848-50.

24 Bal G, David S, Sellier E, et al. Assessment of morbidity and mortality conferences as a tool for physician education and improvement of quality of care and patient safety: a literature review. Presse Med 2010;39:161-8.

25 Sellier E, David-Tchouda S, Bal G, et al. Morbidity and mortality conferences: their place in quality assessments. Int J Health Care Qual Assur 2012;25: 189-96.

26 Handler JA, Gillam M, Sanders AB, et al. Defining, identifying, and measuring error in emergency medicine. Acad Emerg Med 2000;7:1183-8.

27 Seigel TA, McGillicuddy DC, Barkin AZ, et al. Morbidity and Mortality conference in Emergency Medicine. J Emerg Med 2010;38:507-11.

28 Bal G, Sellier E, Tchouda SD, et al. Improving quality of care and patient safety through Morbidity and Mortality Conferences.J Healthc Qual 2012 Apr 24. doi: 10.1111/j.1945-1474.2011.00203.x. [Epub ahead of print].

29 Kauffmann RM, Landman MP, Shelton J, et al. The use of a multidisciplinary morbidity and mortality conference to incorporate ACGME general competencies. J Surg Educ 2011;68:303-8.

30 Hobgood C, Weiner B, Tamayo-Sarver JH. Medical error identification, disclosure, and reporting: do emergency medicine provider groups differ? Acad Emerg Med 2006;13:443-51

31 Leape LL. Reporting of adverse events. N Engl J Med 2002;347:1633-8.

32 Brubacher JR, Hunte GS, Hamilton L, et al. Barriers to and incentives for safety event reporting in emergency departments. Healthc Q 2011;14:57-65. 